\title{
An Efficient Routing Protocol under Noisy Environment for Mobile Ad Hoc Networks using Fuzzy Logic
}

\author{
Supriya Srivastava ${ }^{1}$ and A. K. Daniel ${ }^{2}$ \\ ${ }^{1,2}$ Computer Science \& Engineering Department M M M Engineering College, Gorakhpur U.P. (India)
}

\begin{abstract}
A MANET is a collection of mobile nodes communicating and cooperating with each other to route a packet from the source to their destinations. A MANET is used to support dynamic routing strategies in absence of wired infrastructure and centralized administration. In this paper, we propose a routing algorithm for the mobile ad hoc networks based on fuzzy logic to discover an optimal route for transmitting data packets to the destination. This protocol helps every node in MANET to choose next efficient successor node on the basis of channel parameters like environment noise and signal strength. The protocol improves the performance of a route by increasing network life time, reducing link failure and selecting best node for forwarding the data packet to next node.
\end{abstract}

Keywords-Fuzzy Logic; Noise; Signal Strength; MANET

\section{INTRODUCTION}

Mobile ad hoc network is a collection of mobile devices which can communicate through wireless links. The task of routing protocol is to direct packets from source to destination. This is particularly hard in mobile ad hoc networks due to the mobility of the network elements and lack of centralized control. Source routing is a routing technique in which the sender of a packet determines the complete sequence of nodes through which it forwards the packet. The sender explicitly lists this route in the packet's header, identifying each forwarding "hop" by the address of the next node to which to transmit the packet on its way to the destination host. When a host needs a route to another host, it dynamically determines one based on cached information and on the results of a route discovery protocol, unlike conventional routing protocols.

Routing in MANET using the shortest hop count is not a sufficient condition to construct high-quality routes, because minimum hop count routing often chooses routes that have significantly less capacity than the best routes that exist in the network [1]. The routes selected based on hop count alone may be of bad quality since the routing protocols do not ignore weak quality links which are typically used to connect to remote nodes. The weak quality of a link can be the result of under considered metrics like Energy, SNR, Packet Loss, Maximum available bandwidth, load etc. The links usually have poor signal-to-noise ratio (SNR), hence higher frame error rates and lower throughput [2] [3].

All real measurements in any network are disturbed by noise. This includes electronic noise, but can also include external events that affect the measured phenomenon-wind, vibrations, gravitational attraction of the moon, variations of temperature, variations of humidity, etc., depending on its measurement and of the sensitivity of the device. It is not possible to reduce the noise by controlling the environment.

Otherwise, when the characteristics of the noise are known and are different from the signals, it is possible to filter it or to process the signal. In MANET, it is always needed to choose a channel with lower noise that result in reduction of number of dropped packets to increase the quality of service. The possibility that a packet will drop due to poor signal of the assigned transmission channel is called as "Dropped Call". The dropped packet rate is dependent on the following factors:

- The Channel Capacity

- Level of Traffic in the system

- Probability that noise is above unavoidable frequency.

- Probability that Residual Energy of nodes is below threshold

- Probability that Signal Strength is below Receiver threshold.

- Probability that Signal is below the specified CoChannel interference level.

The proposed approach is called An Efficient Routing Protocol under Noisy Environment for Mobile Ad Hoc Networks using Fuzzy Logic (ERPN). In this paper, the proposed protocol enhances Dynamic Source Routing protocol by considering Signal strength and Noise constraints to improve its performance. The fuzzy logic based technique uses two important parameters as noise factor and signal strength for route selection that results in best possible combinations to choose a route. The proposed protocol defines how a fuzzy logic based technique is effective to select routes, avoid link failure and increase network lifetime. A control mechanism like fuzzy logic is used to make mobile nodes intelligent. Fuzzy logic is basically the extension of crisp logic that includes the intermediate values between absolutely true and absolutely false. It has the efficiency to solve the system uncertainties.

The rest of the paper is organized as follows: related work and design issue in Section 2, the proposed protocol in Section 3 , validation and analysis in Section 4 and finally Conclusion in Section 5. 


\section{DESIGN ISSUES AND RELATED WORK}

The movement of the nodes, packet collision and bad channel condition are the various reasons for the data packets to loss. Packet losses are subjected to occur due to continuous period of intermittent failure during the communication between nodes. The fading conditions cause certain nodes to completely lose their connectivity. Signal-to-Noise ratio is an important issue over a link; the links that usually have bad signal-to-noise ratio (SNR) have higher frame error rates and lower throughput, resulting in link failure. Signal-to-noise ratio (SNR or $\mathrm{S} / \mathrm{N}$ ) compares the level of a desired signal to the level of background noise [4].

- SNR is defined as the ratio of signal power to the noise power. A ratio higher than 1:1 indicates more signal than noise.

$$
S N R=\frac{P_{\text {Signal }}}{P_{\text {noise }}}
$$

Where $P$ is average power

- Both signal and noise power must be measured at the same and equivalent points in a system, and within the same system bandwidth. If the signal and the noise are measured across the same impedance, then the SNR can be obtained by calculating the square of the amplitude ratio:

$$
S N R=\frac{P_{\text {Signal }}}{P_{\text {noise }}}=\left(\frac{A_{\text {signal }}}{A_{\text {noise }}}\right)^{2}
$$

Where $A$ is root mean square (RMS) of amplitude

- In decibels, the SNR is defined as

$$
\begin{aligned}
S N R_{d B} & =10 \log _{10}\left(\frac{P_{\text {Signal }}}{P_{\text {noise }}}\right) \\
& =P_{\text {signal }, d B}-P_{\text {noise }}, d B
\end{aligned}
$$

- $\quad$ Noise Limited System, $\mu \rightarrow 0$

Here, we are considering only noise limited system, so the effect of receiver threshold signal can be considered and also assumed that there will not be any Co-Channel interference. In such a case, $\boldsymbol{\mu} \rightarrow \mathbf{0}$ and the expression for dropped packet rate is

$$
\mathrm{D}=\sum_{n=0}^{N} a_{n} D_{n}=\sum_{n=0}^{N} a_{n}\left[1-(1-\delta)^{\mathrm{n}}\right]
$$

\section{- Interference Limited System, $\delta \rightarrow 0$}

Here, we are consider only interference-limited system, so the effect of Co-Channel interference can be considered and also assumed that there will not be any kind of noise which is introducing in the system. In such a case, $\boldsymbol{\delta} \rightarrow \mathbf{0}$ and the expression for dropped packet rate is

$$
\mathrm{D}=\sum_{n=0}^{N} a_{n} D_{n}=\sum_{n=0}^{N} a_{n}\left[1-(1-\mu)^{\mathrm{n}}\right]
$$

Many algorithms have been proposed for route selection in Mobile Ad-Hoc network in recent years. Some of them are:

Devi M. [5] propose a fuzzy based route recovery technique. It consists of two phases, Proactive failure discovery, and Route failure recovery. Nodes in the network estimate the metrics Link Expiration Time (LET), Received Signal Strength (RSS), Available Band Width (ABW) and Residual Energy (RE) and using fuzzy logic, the type of node is estimated as weak, normal or strong.

Fuad Alnajjar et al. [4] has proposed a mechanism to provide an efficient QoS routing protocol to enhance the performance of existing routing protocols in Mobile ad hoc network environment.

Supriya Srivastava et al [6] proposes an Energy-Efficient Routing protocol that will improve the utilization of link by balancing the energy consumption between utilized and underutilized nodes. It also proposed a method for maintenance of the route during a link failure.

Junghwi Jeon et al. [7] have proposed a fast route recovery scheme to solve the link failure problem caused by node movement, packet collision or bad channel condition.

Merlinda Drini [8] explained that the mobility of the nodes, packet collision and bad channel condition are the various reasons for the data packets to fail. Packet losses are subjected to occur due to continuous period of intermittent failure during the communication between nodes. The fading conditions cause certain nodes to completely lose their connectivity.

Nityananda Sarma et al. [9] have proposed a simple model for computing link stability and route stability based on received signal strengths.

Tomonori Kagi et al. [10] have proposed a reliability improvement method in mobile ad hoc networks by applying network coding encoded by a relay node. Therefore, reliability is improved without requiring the source node to send redundant encoding packets.

Srinivas Sethi et al [11] have proposed an Optimized Reliable Ad hoc On-demand Distance Vector (ORAODV) scheme that offers quick adoption to dynamic link conditions, low processing and low network utilization in ad hoc network.

V. Ramesh et al [12] have proposed a dynamic source routing protocol in which the mobile node uses signal power strength from the received packets to predict the link breakage time, and sends a warning to the source node of the packet if the link is soon-to-be-broken.

Khalid Zahedi et al [13] have proposed and implemented a new approach for solving the problem of link breakages in MANET in Dynamic Source Routing (DSR) routing protocol.

Senthilkumar Maruthamuthu et al [14] have discussed the new protocol QPHMP-SHORT with multiple QoS 
constraints based on the QoS parameters namely delay, jitter, bandwidth, and cost metrics between source and destination.

\section{PROPOSED MODEL For NODE SELECTION USING FUZZY LOGIC BASED TECHNIQUE}

The proposed protocol uses Fuzzy based decision making technique to verify the status of a node. As an outcome of fuzzy decision rules, the node status can be considered as Little Strong, Strong, Very Strong, Lower Medium, Medium, Higher Medium, Little Weak, Weak, and Very Weak. Before a node transmits the data to the next node, it checks the status of that node. This estimated decision is stored in a routing table and is exchanged among all neighbors using a status flag with RREQ message. Data packets are transmitted through intermediate nodes that are in the routing table, whenever the source node sends data to the destination. If the status of a node is Little Weak, Weak or Very Weak then the sending does not transmit the packet to that node, if the status of a node is Lower Medium, Medium or Higher then that node is considerable for receiving the packet from sender node but if the status of a node is Little Strong, Strong, Very Strong then the sending node will choose this node for efficient data packet transmission. The process of node selection consists of two input functions that transform the system inputs into fuzzy sets such as Noise Factor and Signal Strength of paths between any two nodes. Fuzzy set for Noise Factor and Signal Strength in the protocol can be defined as,

And

$$
A=\left\{\left(d, \mu_{A}(n)\right)\right\}, n \in N_{s}
$$

$$
B=\left\{\left(e, \mu_{B}(s)\right)\right\}, s \in S_{i}
$$

Where,

$\mathrm{N}_{\mathrm{s}}$ are universe of discourse for Noise and $\mathrm{S}_{\mathrm{i}}$ is a universe of discourse for Signal Strength,

$\mathrm{n}$ and $\mathrm{s}$ are particular elements of $\mathrm{N}_{\mathrm{s}}$ and $\mathrm{S}_{\mathrm{i}}$ respectively, $\mu_{\mathrm{A}}(\mathrm{n}), \mu_{\mathrm{B}}$ (s) are membership functions, find the degree of membership of the element in a given set.

Membership functions for Noise and Signal Strength are defined from Figure 1, as follows:

$$
\begin{aligned}
\mu_{\mathrm{A}}(\mathrm{n})=\left\{\begin{array}{ll}
0, & \text { if } \mathrm{n}>\mathrm{TH}_{2} \\
\left(\mathrm{n}-\mathrm{TH}_{1}\right) / \mathrm{TH}_{1}-\mathrm{TH}_{2}, & \text { if } \mathrm{TH}_{1} \geq \mathrm{n} \geq \mathrm{TH}_{2} \\
1, & \text { if } \mathrm{n} \leq \mathrm{TH}_{1}
\end{array}\right\} \\
\mu_{\mathrm{B}}(\mathrm{s})=\left\{\begin{array}{ll}
0, & \text { if } \mathrm{s} \leq \mathrm{TH}_{1} \\
\left(\mathrm{TH}_{1}-\mathrm{s}\right) / \mathrm{TH}_{1}-\mathrm{TH}_{2}, & \text { if } \mathrm{TH}_{1}<\mathrm{s}<\mathrm{TH} H_{2} \\
1, & \text { if } \mathrm{s} \geq \mathrm{TH}_{2}
\end{array}\right\}
\end{aligned}
$$

Where,

$\mathrm{TH}_{1}=$ Threshold to activate system

$\mathrm{TH}_{2}=$ Threshold which identifies the level of activeness

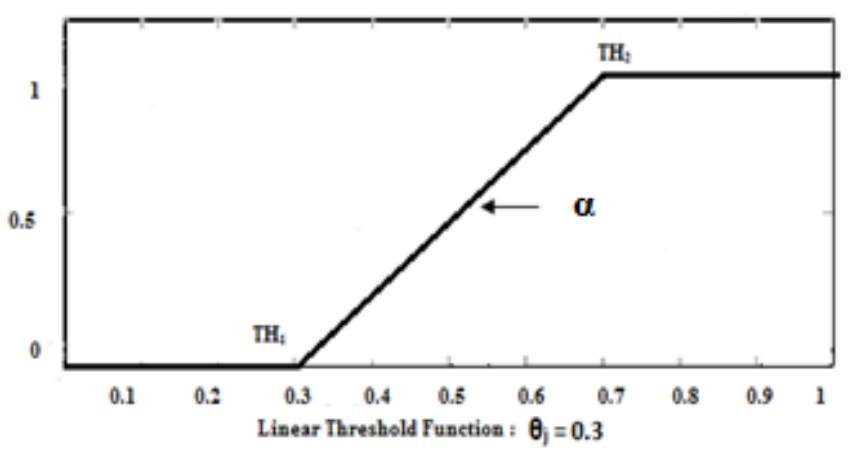

Fig. 1. Graph showing minimum and maximum threshold for any input variable

A fuzzy relation is a relation between elements of $\mathrm{N}_{\mathrm{s}}$ and elements of $S_{i}$, described by a membership function,

$$
\mu_{\mathrm{Ns} X \mathrm{Si}}(\mathrm{n}, \mathrm{s}), \mathrm{n} € \mathrm{~N}_{\mathrm{s}} \text { and } \mathrm{s} € \mathrm{~S}_{\mathrm{i}}
$$

Now applying AND fuzzy operator i.e. min $(\boldsymbol{\Lambda})$ on fuzzy relation,

$$
\begin{aligned}
& \boldsymbol{\mu}_{A}(\mathrm{n}) \Lambda \boldsymbol{\mu}_{B}(\mathrm{~s})=\min \left(\mu_{A}(n), \mu_{B}(s)\right) \\
& \quad=\left\{\begin{array}{ll}
\mu_{A}(n), & \text { if and only if } \mu_{A}(n) \leq \mu_{B}(s) \\
\mu_{B}(s), & \text { if and only if } \mu_{A}(n) \geq \mu_{B}(s)
\end{array}\right\}
\end{aligned}
$$

\section{A. Rule Evaluation}

The proposed protocol is a fuzzy logic based protocol for the selection of successor node for data packet transmission. The process of route selection consists of two input functions that transform the system inputs into fuzzy sets such as Noise Factor and Signal Strength of paths between any two nodes.

The Table 1 of Input Function uses three membership functions to show the varying degrees of input variables.

TABLE I. INPUT FUNCTION

\begin{tabular}{|c|c|c|c|}
\hline Input & \multicolumn{3}{|c|}{ Membership } \\
\hline Noise Factor & Light & Medium & Heavy \\
\hline Signal Strength & Weak & Adequate & Strong \\
\hline
\end{tabular}

In Table 2, 9 membership functions are defined that represent the varying output memberships of the fuzzy output defined for each of the rules in the rule set and the graph for the same is shown in Figure 2.

Then an aggregation of these fuzzy probabilistic values into a single fuzzy output is represented in a detailed rule-set (Table 3).

TABLE II. OUTPUT FUNCTION

\begin{tabular}{|c|l|}
\hline Output & \multicolumn{1}{|c|}{ Membership } \\
\hline Output & Little Strong, Strong, Very Strong, Lower \\
Memberships & $\begin{array}{l}\text { Medium, Medium, Higher, Medium, Little } \\
\text { Weak, Weak, Very Weak }\end{array}$ \\
\hline
\end{tabular}




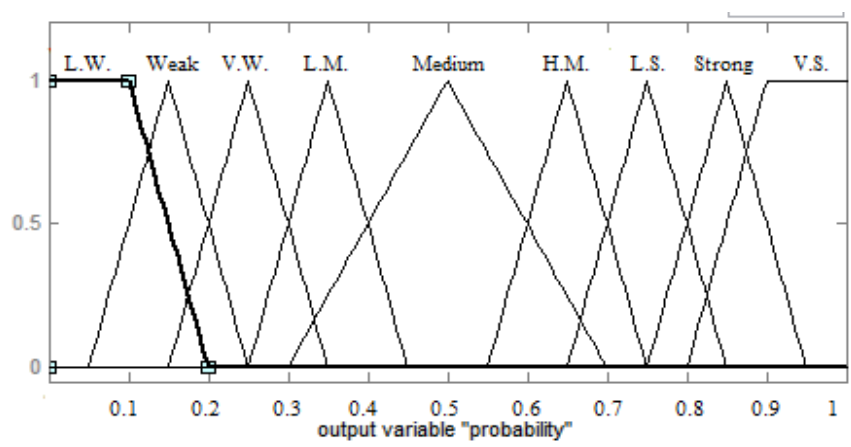

Fig. 2. Graph showing all possible probabilities of output variable

\section{B. The Proposed Rule set}

The given protocol defines all the possible combinations of the different membership functions for the two input variables that results in 9 rules for the fuzzy inference shown in Table 3.

TABLE III. RULE SET TABLE

\begin{tabular}{|c|c|c|}
\hline Noise Factor & Signal Strength & Output Memberships \\
\hline Light & Weak & Higher Medium \\
\hline Light & Adequate & Strong \\
\hline Light & Strong & Very Strong \\
\hline Medium & Weak & Little Weak \\
\hline Medium & Adequate & Medium \\
\hline Medium & Strong & Little Strong \\
\hline Heavy & Weak & Very Weak \\
\hline Heavy & Adequate & Weak \\
\hline Heavy & Strong & Lower Medium \\
\hline
\end{tabular}

Rule 1

IF Noise Factor is Light AND Signal Strength is Weak

THEN output membership is Higher Medium

Rule 2

IF Noise Factor is Light AND Signal Strength is Adequate

THEN output membership is Strong

Rule 3

IF Noise Factor is Light AND Signal Strength is Strong THEN output membership is Very Strong

Rule 4

IF Noise Factor is Medium AND Signal Strength is Weak THEN output membership is Little Weak

Rule 5

IF Noise Factor is Medium AND Signal Strength is Adequate THEN output membership is Medium

Rule 6

IF Noise Factor is Medium AND Signal Strength is Strong THEN output membership is Little Strong

Rule 7

IF Noise Factor is Heavy AND Signal Strength is Weak THEN output membership is Very Weak

Rule 8

IF Noise Factor is Heavy AND Signal Strength is Adequate THEN output membership is Weak

Rule 9

IF Noise Factor is Heavy AND Signal Strength is Strong THEN output membership is Lower Medium

\section{VALIDATION AND ANALYSIS}

Let us consider a network of 5 nodes and 6 edges. Now suppose the noise factor at each edge as:

$$
\text { Ns }=\{0.1,0.3,0.4,0.7,0.8,0.9\}
$$

And the signal strength at each edge as:

$$
\mathrm{Si}=\{0.2,0.3,0.5,0.6,0.9,01\}
$$

A membership function based on the noise at each edge and its graphical representation is shown in Figure-3

$$
\mu_{\text {Noise }}(n)=\left\{\begin{array}{ll}
0, & \text { if }_{\text {Noise }}(\mathrm{n}) \geq 0.8\left(\mathrm{TH}_{2}\right) \\
(0.8 \text {-Noise }(\mathrm{n})) / 0.2, & \text { if } 0.6<\text { Noise }(\mathrm{n})<0.8 \\
1, & \text { if }_{\text {Noise }}(\mathrm{n}) \leq 0.6
\end{array}\right\}
$$

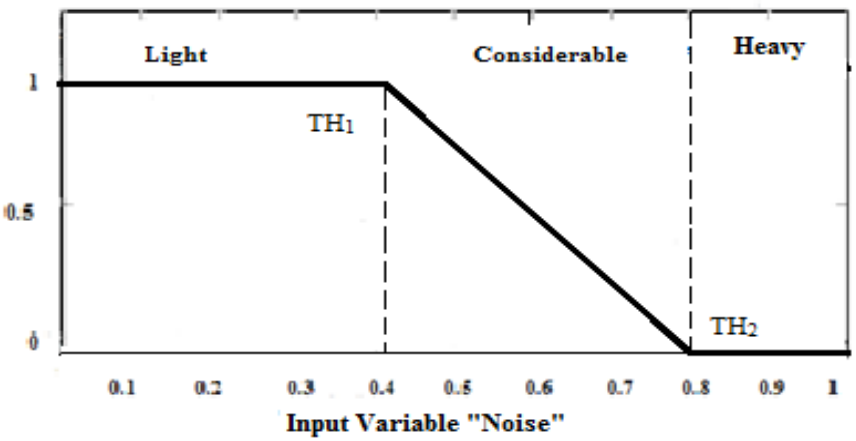

Fig. 3. Graph showing membership functions for input variable "Noise"

A membership function based on the Signal Strength at each edge and its graphical representation is shown in Figure4

$\mu_{\text {Signal }}(\mathrm{s})=\left\{\begin{array}{ll}0, & \text { if } \text { Signal }(\mathrm{s}) \leq 0.3\left(\mathrm{TH}_{1}\right) \\ \left(\text { Signal }_{1}(\mathrm{~s})-0.3\right) / 0.4, & \text { if } 0.3<\text { Signal }(\mathrm{s})<0.7 \\ 1, & \text { if } \text { Signal }_{\text {S }}(\mathrm{s}) \geq 0.7\end{array}\right\}$

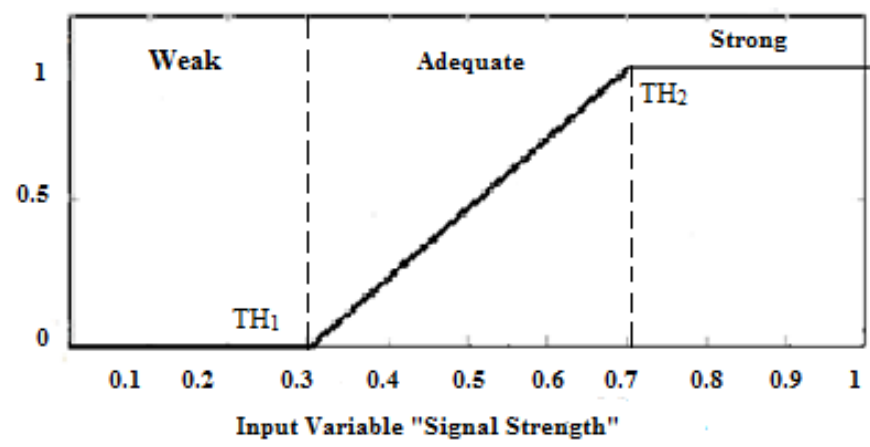

Fig. 4. Graph showing membership functions for input variable "Signal Strength"

Now calculate the degree of membership of Noise and Signal Strength using the above defined membership functions for both of these input variables which are shown in Table 4 and Table 5 . 
TABLE IV. DEGREE OF MEMBERSHIP OF NOISE

\begin{tabular}{|l|l|}
\hline Noise (Ns) & Degree of Lightness \\
\hline 0.1 & 1 \\
\hline 0.3 & 1 \\
\hline 0.4 & 1 \\
\hline 0.7 & 0.5 \\
\hline 0.8 & 0 \\
\hline 0.9 & 0 \\
\hline
\end{tabular}

In Table 4 for the Noise factors $\{0.1,0.3,0.4,0.7,0.8$, $0.9\}$ the degree of memberships are $\{1,1,1,0.5,0,0\}$ respectively. According to fuzzy output the memberships of the above noise factors are,

\{0.1| weak, 0.3| weak, 0.4| weak, 0.7|Considerable, 0.8| Considerable, 0.9| heavy\}

TABLE V. DEGREE OF MEMBERSHIP OF SIGNAL STRENGTH

\begin{tabular}{|l|l|}
\hline Signal (Si) & Degree of Strongness \\
\hline 0.2 & 0 \\
\hline 0.3 & 0 \\
\hline 0.5 & 0.5 \\
\hline 0.6 & 0.75 \\
\hline 0.9 & 1 \\
\hline 01 & 1 \\
\hline
\end{tabular}

In Table 5 for the Signal Strengths $\{0.2,0.3,0.5,0.6,0.9$, $1\}$ the degree of memberships are $\{0,0,0.5,0.75,1,1\}$ respectively. According to fuzzy output the memberships of the above Signal Strengths are,

$\{0.2 \mid$ weak, 0.3| weak, 0.4| Adequate, 0.7| Adequate, 0.8 Strong, 0.9| Strong\}

Now create Table 6 that shows the fuzzy relation between membership functions of noise factor and signal strength.

TABLE VI. FUZZY RELATION ON MEMBERSHIP VALUE OF SIGNAL STRENGTH AND NOISE

\begin{tabular}{|c|c|c|c|c|c|c|}
\hline$\frac{S i}{N s}$ & 0.2 & 0.3 & 0.5 & 0.6 & 0.9 & 1 \\
\hline 0.1 & $1 \Lambda 0$ & $1 \Lambda 0$ & $1 \Lambda 0.5$ & $1 \Lambda 0.75$ & $1 \Lambda 1$ & $1 \Lambda 1$ \\
\hline 0.3 & $1 \Lambda 0$ & $1 \Lambda 0$ & $1 \Lambda 0.5$ & $1 \Lambda 0.75$ & $1 \Lambda 1$ & $1 \Lambda 1$ \\
\hline 0.4 & $1 \Lambda 0$ & $1 \Lambda 0$ & $1 \Lambda 0.5$ & $1 \Lambda 0.75$ & $1 \Lambda 1$ & $1 \Lambda 1$ \\
\hline 0.7 & $0.5 \Lambda 0$ & $0.5 \Lambda 0$ & $0.5 \Lambda 0.5$ & $0.5 \Lambda 0.75$ & $0.5 \Lambda 1$ & $0.5 \Lambda 1$ \\
\hline 0.8 & $0 \boldsymbol{\Lambda} 0$ & $0 \Lambda 0$ & $0 \Lambda 0.5$ & $0 \Lambda 0.75$ & $0 \Lambda 1$ & $0 \Lambda 1$ \\
\hline 0.9 & $0 \Lambda 0$ & $0 \boldsymbol{\Lambda} 0$ & $0 \Lambda 0.5$ & $0 \Lambda 0.75$ & $0 \boldsymbol{\Lambda} 1$ & $0 \Lambda 1$ \\
\hline
\end{tabular}

The result of the AND $(\boldsymbol{\Lambda})$ operation process on membership values of Signal Strength and Noise shown in Table 7.

TABLE VII. RESULT AFTER AND FUZZY OPERATION

\begin{tabular}{|l|l|l|l|l|l|l|}
\hline$\frac{\boldsymbol{S i}}{\boldsymbol{N} \boldsymbol{s}}$ & $\mathbf{0 . 2}$ & $\mathbf{0 . 3}$ & $\mathbf{0 . 5}$ & $\mathbf{0 . 6}$ & $\mathbf{0 . 9}$ & $\mathbf{1}$ \\
\hline $\mathbf{0 . 1}$ & 0 & 0 & 0.5 & 0.75 & 1 & 1 \\
\hline $\mathbf{0 . 3}$ & 0 & 0 & 0.5 & 0.75 & 1 & 1 \\
\hline $\mathbf{0 . 4}$ & 0 & 0 & 0.5 & 0.75 & 1 & 1 \\
\hline $\mathbf{0 . 7}$ & 0 & 0 & 0.5 & 0.5 & 0.5 & 0.5 \\
\hline $\mathbf{0 . 8}$ & 0 & 0 & 0 & 0 & 0 & 0 \\
\hline $\mathbf{0 . 9}$ & 0 & 0 & 0 & 0 & 0 & 0 \\
\hline
\end{tabular}

The possible combinations of distance and energy with higher membership value shown in Table 8:

TABLE VIII. OUTPUT TABLE

\begin{tabular}{|l|l|l|}
\hline$\frac{S i}{N S}$ & 0.9 & 1 \\
\hline 0.1 & 1 & 1 \\
\hline 0.3 & 1 & 1 \\
\hline 0.4 & 1 & 1 \\
\hline
\end{tabular}

The degree of membership of noise and signal is shown in Table 9.

TABLE IX. OUTPUT TABLE WITH THE DEGREE OF MEMBERSHIP

\begin{tabular}{|l|l|l|l|}
\hline $\begin{array}{l}\text { Noise } \\
(\mathrm{Ns})\end{array}$ & $\begin{array}{l}\text { Degree of } \\
\text { membership } \\
\text { (Noise) }\end{array}$ & $\begin{array}{l}\text { Signal } \\
\mathbf{( S i )}\end{array}$ & $\begin{array}{l}\text { Degree } \\
\text { Strongness } \\
\text { (Signal) }\end{array}$ \\
\hline $\mathbf{0 . 1}$ & Light & $\mathbf{0 . 9}$ & Strong \\
\hline $\mathbf{0 . 3}$ & Light & $\mathbf{1}$ & Strong \\
\hline $\mathbf{0 . 4}$ & Light & & \\
\hline
\end{tabular}

The output memberships for these values are "Very Strong" as "Noise is Light and Signal strength is strong". Now all the resultant possible combinations of noise and signal strength are:

- Noise factor $=0.1$ and Signal strength $=0.9$

- Noise factor $=0.1$ and Signal strength $=1$

- $\quad$ Noise factor $=0.3$ and Signal strength $=0.9$

- Noise factor $=0.3$ and Signal strength $=1$

- $\quad$ Noise factor $=0.4$ and Signal strength $=0.9$

- Noise factor $=0.4$ and Signal strength $=1$.

The effective edge to be selected can have any of the above combinations. But the perfect combination among all combinations is when noise factor is 0.1 and signal strength is 1 , which will be perfect for transmission of data packet to next successor node.

\section{A. Performance Evaluation}

The proposed protocol is a fuzzy logic based protocol for the efficient successive edge selection for data packet transmission. The best possible outcomes obtained from the above proposed fuzzy logic based protocol helps a mobile AdHoc network to choose an efficient edge on the basis of parameters like environment noise and signal strength. The protocol improves the performance of a route by increasing network life time, reducing link failure and selecting best node for forwarding the data packet to next node.

\section{CONCLUSIONS}

The proposed protocol an Efficient Routing Protocol under Noisy Environment for Mobile Ad Hoc Networks using Fuzzy Logic (ERPN) is efficient for transmission of data. The status of the node is verified before a node transmits the data to the 
next node. The designed fuzzy logic controller determines, best outcome from all the possible combinations of offered signal strength and noise. If the status is normal or strong, then it transmits the packet to the next node. The validation shows that the fuzzy based effective edge selection technique increases packet delivery ratio, decreases link failure, lowers error rate and increases throughput.

\section{REFERENCES}

[1] Wing Ho Yuen, Heung-no Lee and Timothy D. Andersen "A simple and effective cross layer networking system for Mobile Ad Hoc Networks", in: PIMRC 2002, vol. 4, September 2002, pp. 1952-1956.

[2] H.M. Tsai, N. Wisitpongphan, and O.K. Tonguz, "Link-quality aware AODV protocol," in Proc. IEEE International Symposium on Wireless Pervasive Computing (ISWPC) 2006, Phuket, Thailand, January 2006

[3] E. Royer and C.-K. Toh, "A review of current routing protocols for Ad Hoc mobile wireless networks," IEEE Personal Communications Magazine, vol. 6, No. 2, April 1999.

[4] Fuad Alnajjar and Yahao Chen "SNR/RP aware routing algorithm crosslayer design for MANET" in International Journal of Wireless \& Mobile Networks (IJWMN), Vol 1, No 2, November 2009.

[5] H Devi M. and V. Rhymend Uthariaraj "Fuzzy based route recovery technique for Mobile Ad Hoc Networks" in European Journal of Scientific Research ISSN 1450-216X Vol.83 No.1 (2012), pp.129-143.

[6] Supriya Srivastava, A.K. Daniel "Energy-efficient position based routing protocol for MANET in proceedings of International Conference in computing, engineering and information technology (ICCEIT), 2012.

[7] Junghwi Jeon., Kiseok Lee., and Cheeha Kim., "Fast route recovery scheme for Mobile Ad Hoc Networks", IEEE International Conference on Information Networking, 2011.

[8] Merlinda Drini \& Tarek Saadawi ."Modeling wireless channel for AdHoc network routing protocol", ISCC Marakech Marocco, July 2008, Page(s): 549-555

[9] Nityananda Sarma., and Sukumar Nandi., "Route stability based QoS routing in mobile Ad Hoc networks", IEEE international Conference on Emerging Trends in Engineering and Technology, 2008.

[10] Tomonori Kagi., and Osamu Takahashi., "Efficient reliable data transmission using network coding in MANET multipath routing environment", Springer, KES, 2008.

[11] Srinivas Sethi., and Siba K. Udgata., "Optimized and reliable AODV for MANET", International Journal of Computer Applications, 2010.

[12] V.Ramesh., Dr.P.Subbaiah., and K.Sangeetha Supriya., "Modified DSR (Preemptive) to reduce link breakage and routing overhead for MANET using Proactive Route Maintenance (PRM)", Global Journal of Computer Science and Technology, 2010.

[13] Khalid Zahedi., and Abdul Samad Ismail.,"Route maintenance approach for link breakage predicttion in mobile Ad Hoc networks", International Journal of Advanced Computer Science and Applications ((IJACSA), 2011.

[14] Senthilkumar Maruthamuthu., and Somasundaram Sankaralingam., " QoS aware power and hop count constraints routing protocol with mobility prediction for MANET using SHORT", International Journal of Communications, Network and System Sciences, 2011. 